\title{
Estrategia de enseñanza y aprendizaje de programación basada en la idea de 'hackathon"*
}

\author{
María Piles $^{1}$, Valero Laparra ${ }^{1}$, Adrián Pérez-Suay ${ }^{1}$, Gonzalo Mateo-García ${ }^{1}$, Vicent \\ Girbés-Juan $^{1}$, María Moreno-Llácer ${ }^{1}$ y Jordi Muñoz-Marí ${ }^{1}$ \\ ${ }^{1}$ Universitat de València
}

\begin{abstract}
The acquisition of programming and data analysis skills in higher education is increasingly necessary in all areas of Science and Engineering. In this paper we present a methodology for the motivation of programming learning, mainly focused on the development of machine learning algorithms. This methodology is based on the hackathon idea and will have different levels. On the one hand the basic level where a competition is proposed in an improvised way during the development of the class. A second level where a programmed hackathon is proposed but within the classroom environment and using learning management systems such as Moodle. The last level consists of participation in an external hackathon and the delivery of a report. These levels have been adapted and tested in several undergraduate and master's degree courses at the University of Valencia. We include detailed information on how the methodology has been adapted to the teaching needs of the subject and we conducted anonymized surveys to students to know their degree of satisfaction. These surveys reveal a positive assessment of the experience by the students and include constructive comments for improvement in future editions.
\end{abstract}

Keywords: hackathon, datathon, programming, evaluation, participation, motivation.

\begin{abstract}
Resumen
La adquisición de competencias en programación y análisis de datos en la educación superior es cada vez más necesaria en todas las áreas de las Ciencias e Ingeniería. En este trabajo presentamos una metodología para la motivación del aprendizaje de programación, principalmente enfocada al desarrollo de algoritmos de machine learning. Esta metodología está basada en la idea de hackathon y tendrá distintos niveles. Por un lado el nivel básico donde se plantea una competición de forma improvisada durante el desarrollo de la clase. Un segundo nivel donde se plantea un hackathon programado
\end{abstract}

\footnotetext{
* Proyecto de innovación educativa "Explotación de las herramientas online de la Universitat de València para la evaluación a distancia de asignaturas en el área de ciencia" curso (2020-21) UV-SFPIE_PID-1354708
} 
pero dentro del entorno de la clase y utilizando sistemas de gestión de aprendizaje tipo Moodle. El último nivel consiste en la participación en un hackathon externo y la entrega de un informe. Estos niveles han sido adaptados y testeados en varias asignaturas de grado y máster de la Universitat de València. Incluimos información detallada de cómo se ha adaptado la metodología a las necesidades docentes de la asignatura y realizamos encuestas anonimizadas a los estudiantes para conocer su grado de satisfacción. Dichas encuestas revelan una valoración positiva de la experiencia por parte del alumnado e incluyen comentarios constructivos para la mejora en siguientes ediciones.

Keywords: hackathon, datathon, programación, evaluación, participación, motivación.

\section{Introducción}

La palabra hackathon es una combinación de las palabras "hack" y "marathon" donde hack se refiere a una solución experimental y creativa de problemas con un enfoque lúdico, y marathon se refiere a la duración relativamente corta pero intensa del evento. El hackathon tiene su origen en la ingeniería de software y hardware, pero el concepto también se aplica ahora con éxito en otros campos para desarrollar soluciones innovadoras.

Un hackathon no tiene una duración definida pero suele estar acotado temporalmente, pudiendo durar horas, días o incluso semanas y está dedicado a un tema o reto específico. En lo que se refiere a modalidades, puede ser presencial, lo cual añade una restricción espacial además de la temporal, u online, desarrollándose a través de alguna plataforma de recopilación de datos. Plataformas como Zindi $^{1}$ o Kaggle ${ }^{2}$ son ejemplos donde se plantean hackathons virtuales. Los participantes trabajan en grupos reducidos en un entorno único que fomenta el pensamiento creativo y conduce a nuevos conceptos, ideas y prototipos sorprendentemente innovadores. El resultado del hackathon es un prototipo terminado para un producto, servicio o incluso un modelo de negocio innovador.

Aunque un hackathon está orientado principalmente a desarrolladores, lo cierto es que esta metodología se está extendiendo rápidamente a otros ámbitos, como el educativo (García 2020), y no solamente se celebran hackathones para desarrollar aplicaciones, sino también para otros propósitos, como por ejemplo para encontrar soluciones creativas a problemas de colectivos u organizaciones (Muñoz, Silvente y Viñals 2017).

Un hackathon supone una magnifica experiencia para que los alumnos pongan en práctica las competencias y habilidades con el objetivo de resolver problemas en un contexto real.

Durante un hackathon los participantes deben organizarse en equipos según sus diferentes habilidades con un objetivo común: ¿Cómo podemos resolver de la mejor manera este problema en el tiempo que tenemos a disposición? La propia estructura del evento supone un aliciente que dispara la creatividad y los sprints de diseño son realmente productivos. El ambiente predominante

\footnotetext{
${ }^{1}$ https: //zindi.africa

${ }^{2}$ https://kaggle.com
} 
es distendido, estando la cooperación por encima de la competición: los participantes comparten conocimientos y aprendizajes en un contexto real y rápidamente aplicable.

Si trasladamos esto a un entorno educativo las posibilidades que ofrece esta metodología son rápidamente apreciables: más allá del aprendizaje y la puesta en práctica de habilidades para resolver problemas en contextos reales, los alumnos tienen no sólo una magnífica oportunidad para aprender y utilizar técnicas de design thinking, aprendizaje basado en proyectos, trabajo colaborativo y habilidades de comunicación, sino también la posibilidad de abordar un proyecto desde el enfoque STEM (por sus siglas en inglés: Science, Technology, Engineering and Maths) en el que las Ciencias, la Tecnología, la Ingeniería y las Matemáticas se trabajan como una sola disciplina permitiendo profundizar en la esencia del problema y en las necesidades que éste plantea (Dolgopolovas y Dagienė 2021). En el trabajo Porras y col. 2018 los autores presentan una taxonomía de los diferentes tipos de hackatons. Entre los hackathones para educación, éstos no están restringidos a niveles universitarios, sinó que también pueden utilizarse para fomentar las competencias digitales en las escuelas Dorn y col. 2020. En Gardó y Riera 2020 los autores utilizan cuestionarios y entrevistas para investigar el uso de hackathones educativos y concluyen que las soluciones obtenidas en éstos se valoran por la libertad del proceso creativo, la generación de redes sociales y el aprendizaje social en la creación de las soluciones.

En este trabajo se presenta una estrategia de enseñanza y aprendizaje de programación basada en la idea de 'hackaton'. La metodología propuesta se desarrolla y analiza con casos prácticos de las asignaturas y titulaciones que se detallan en la tabla Tabla 1.

Tabla 1: Asignaturas analizadas en el estudio de diversas titulaciones de la Universitat de València.

\begin{tabular}{|c|c|c|c|}
\hline Titulación & Asignatura & Curso & № alumnos \\
\hline Máster en inteligencia artificial & Aprendizaje profundo & $1^{\mathrm{o}}$ & $20-30$ \\
\hline Máster en Teledetección & Análisis y Extracción de la información & $1^{\mathrm{O}}$ & $15-25$ \\
\hline Máster en ciencia de datos & Aprendizaje máquina II & $1^{\mathrm{O}}$ & $20-30$ \\
\hline Grado en ciencia de datos & Aprendizaje máquina & $3^{\mathrm{o}}$ & $20-30$ \\
\hline Grado en Ingeniería Electrónica Industrial & Control digital & $3^{\mathrm{o}}$ & $15-20$ \\
\hline Máster en ciencia de datos & Big Data & $1^{\mathrm{o}}$ & $25-35$ \\
\hline
\end{tabular}

\section{Objetivos}

El objetivo de la metodología docente que proponemos en esta comunicación es la motivación del aprendizaje de programación, una competencia transversal en muchas titulaciones técnicas en la que es fundamental la experiencia práctica, y que requiere un número relativamente alto de horas de dedicación (Mohan y col. 2010). La metodología propuesta está principalmente enfocada al desarrollo de algoritmos de aprendizaje máquina, competencia central en titulaciones como el grado en ingeniería electrónica, el grado en ciencia de datos, el máster de ciencia de datos y el máster de inteligencia artificial y aplicada de la Universitat de València (UV). No obstante, la 
mayoría de las metodologías planteadas son susceptibles de ser utilizadas en otras materias que requieran trabajar con los elementos básicos de los lenguajes de programación para planteamiento y resolución de problemas.

Los objetivos generales están centrados en resolver los siguientes problemas intrínsecos en la docencia de la programación informática:

- Motivación a programar. La clase magistral cómo metodología docente puede no ser la más adecuada para enseñar a programar, aunque si es necesario conocer la sintaxis y elementos que la componen (bucles, tipos de datos, etc.) un enfoque más práctico es el que permite aprender a programar programando. Por ello es necesario encontrar metodologías que animen al alumno a programar y, sobre todo, a aprovechar las horas de práctica dentro de las clases.

- Romper el ritmo de fatiga. Normalmente las clases de programación constan de dos o más horas, además son especialmente tediosas debido a la densidad de las mismas. Por ello es necesario desarrollar metodologías que rompan el ritmo de fatiga (Imbernon 2014) ayudando a dinamizar.

- Plantear problemas verosímiles y reales Un mantra repetido por los estudiantes es que al acabar sus estudios universitarios no sabrían cómo aplicar sus conocimientos en el mundo real. Nuestra idea es intentar paliar este problema enfrentando a los alumnos a casos prácticos verosímiles o directamente reales (planteados en una competición existente).

El hackathon ayuda a mejorar la motivación a programar y dinamiza la clase rompiendo el ritmo de fatiga, además de permitir plantear problemas verosímiles $y$ reales que se resuelven de forma colaborativa.

Para conseguir estos objetivos planteamos metodologías a 3 niveles diferentes. Los niveles están numerados de más sencillo a más complejo de aplicar:

- Nivel 1. Proponer una metodología de aplicación simple de hackathon. Se trata de una estrategia que ayude a romper el ritmo de fatiga de la sesión, por lo que tiene que ser dinámica, y a su vez motive a programar al alumno. Tiene que ser directa y de aplicación inmediata.

- Nivel 2. Proponer una metodología que permita hacer una evaluación interna utilizando algún sistema de gestión de aprendizaje. Dentro de las técnicas de motivación la más directa es la evaluación. Por tanto, proponemos modelos de hackathon interno que por un lado nos permitan su planteamiento de una forma más o menos dinámica, pero que a su vez asegure una evaluación de los resultados justa. Además, esta metodología permitirá plantear un entorno realista.

- Nivel 3. Proponer una metodología que implique un reto real. Es necesario tener una herramienta que permita enfrentar a los alumnos a retos que ellos conciban como reales, es decir, que sean iguales a los que se enfrentarán una vez terminen los estudios. Para ello, por un lado la evaluación (o una parte) ha de ser externa, es decir, no es el docente quien evalúa el trabajo sino alguien ajeno al docente y a los alumnos, y por otro lado el reto no ha de estar 
restringido al ámbito de la clase. Normalmente una evaluación tiene parte de competición interna, ya que las notas de los alumnos tienden a compararse entre ellos, la idea es romper esta dinámica haciendo que los alumnos no sólo compitan entre ellos, sino que lo hagan con más personas.

\section{Desarrollo de la innovación}

Se propone que las clases combinen la parte teórica con la práctica sin distinción entre sesiones dedicadas a teoría y práctica. Se distinguen tres niveles de aplicación de la metodología directamente relacionados con los objetivos marcados (sec. 2). Un primer nivel consiste en realizar en una primera parte de la sesión un desarrollo expositivo de la materia y en una segunda parte plantear una competición a modo de gamificación para motivar la participación del estudiante en la resolución de cuestiones puntuales. En este nivel se valora la participación como parte de la evaluación continua y no se realiza ninguna entrega. Un segundo nivel consiste en plantear un hackathon programado dentro del entorno de la clase y utilizando Moodle. Esta actividad puede ser una tarea para evaluar un bloque temático, o también se puede diseñar como examen final de la asignatura. En el último caso la participación deberá ser individual. El tercer nivel consiste en plantear la participación por equipos (2-3 personas) en un hackathon externo y la entrega de un informe, a través del cual se evalúan las competencias adquiridas en los diferentes aspectos de la materia y se fomenta el trabajo en equipo. En las siguientes secciones se proporcionan casos prácticos de aplicación de los tres niveles de la metodología propuesta.

\subsection{Nivel 1}

\subsubsection{Aprendizaje Profundo y Aprendizaje Máquina II}

Uno de los principales problemas en las asignaturas de muchos estudios de máster es que se tiende a concentrar las temáticas de modo que se aumenta la longitud de las sesiones. En el máster de ciencia de datos de la Universitat de València las clases suelen tener una duración de 2 horas y media y en el máster de inteligencia artificial suelen ser de 5 horas. Esto hace que sean imprescindibles técnicas de ruptura del ritmo de fatiga (Imbernon 2014).

En la asignatura de aprendizaje profundo impartida en el máster de inteligencia artificial y la asignatura Aprendizaje máquina II en el master de ciencia de datos hemos empleado la metodología del hackathon para romper la fatiga obteniendo unos muy buenos resultados y un alto nivel de participación. El procedimiento es muy práctico porque es muy fácilmente implementable.

- Después de la explicación de una metodología de aprendizaje profundo se propone a los alumnos una competición, de manera individual o por parejas. Se recomienda que la competición parta de un ejemplo ya resuelto por el docente. A los alumnos se les propone modificar el modelo propuesto por el docente de forma que maximicen una función objetivo. En el caso del aprendizaje profundo dividiremos los datos en dos subconjuntos (entrenamiento y test); los alumnos desarrollarán su modelo con los datos de entrenamiento y reportarán sus resultados en los datos de test.

- Mientras los alumnos empiezan su modificación se crea una tabla de datos en cualquier programa de hojas de cálculo (excel, google docs...). Es importante que esta tabla esté visible 
por los alumnos en todo momento, por ejemplo, mostrándola por el proyector, de esta forma motiva más ver los resultados de los compañeros comparados directamente con los propios. También es importante que a los alumnos les quede claro que por un lado la participación es obligatoria pero por otro los resultados no son importantes. Es decir, en esa tabla tiene que haber un resultado de cada alumno, pero que no se va a evaluar el rendimiento del modelo sino la participación.

- Conforme los alumnos van obteniendo resultados el docente los va colocando en la tabla. Aunque la tabla se haga en una plataforma que permita la colaboración, es importante que sólo el docente tenga acceso a modificar esta tabla (el caso contrario distrae a los alumnos). Ver los resultados de los compañeros motiva a la participación y la competición. Si el tiempo lo permite es interesante que cada alumno explique brevemente no sólo su resultado sino cuál ha sido el procedimiento. Esto permite al profesor remarcar tanto cosas positivas como cosas negativas.

- Al final hay una puesta en común. Se toman los métodos con mejores resultados y se pide a los alumnos que los han obtenido que expliquen brevemente qué piensan que ha sido lo que ha hecho que su método funcione. Esto motiva a otros alumnos que han hecho cosas similares y no les ha funcionado a preguntar y contrastar las metodologías.

\subsubsection{Análisis y Extracción de la Información}

En la asignatura Análisis y Extracción de la Información (Máster en teledetección) pusimos en práctica el nivel 1 mediante actividades concretas en las que invitábamos a los alumnos a que se enfrentaran activamente a un problema planteado. Los alumnos tenían conocimientos previos de métodos estadísticos de regresión que habíamos trabajado en las sesiones anteriores, así como de procesado e interpretación de datos satelitales espacio-temporales (Camps-Valls y col. 2011).

El problema propuesto en una sesión que incluimos aquí de ejemplo fue la estimación del índice de área folial (Leaf Area Index, LAI) a partir de las 64 bandas espectrales disponibles de una imagen del sensor Compact High Resolution Imaging Spectrometer (CHRIS), abordo del satélite Proba-1. La zona de estudio era Barrax, Albacete, donde se tomaron medidas de campo LAI al tiempo de paso del satélite y que también se proporcionan para entrenar y validar los modelos (Verrelst y col. 2012). Al principio de esta sesión trabajamos la demo (un script informático) donde secuencialmente se cargan los datos, se visualizan, y se dividen en dos grupos, el de entrenamiento y el de test. Como ejemplo, se muestra el desarrollo de un modelo paramétrico de regresión que usa dos bandas espectrales como entrada para la estimación de LAI. Se realiza primero un entrenamiento del modelo con los datos de entrenamiento y luego se aplica el modelo entrenado a los datos de test y se obtienen unas métricas de validación. En esta primera fase de entendimiento del problema, ejecutamos paso a paso la demo entre todos, donde cada alumno contribuía con la interpretación de una línea del código proporcionado. En caso de dudas se les invitó a utilizar la palabra clave 'pasapalabra' para que respondiera el siguiente compañero. Una vez ejecutada la demo entre todos, creamos equipos de 2-3 personas, y a cada equipo se le propuso el uso de un método paramétrico o no paramétrico (de la Tabla 6.1 de Camps-Valls y col. 2011) para la estimación del parámetro LAI. En la pizarra creamos una tabla para registrar las métricas de validación obtenidas con el conjunto de datos de test. Les dimos 20 minutos para realizar la actividad, durante los cuales fuimos resolviendo dudas y apuntando en la pizarra los resultados. En la última parte de la sesión comentamos entre todos los resultados obtenidos, ventajas y desventajas de las diferentes aproximaciones. 


\subsection{Nivel 2}

\subsubsection{Aprendizaje máquina}

En la asignatura de aprendizaje máquina (Grado de ciencia de Datos) pusimos en práctica el nivel 2 de dos formas distintas.

Primer procedimiento: en prácticas. Primero planteamos un hackathon interno en una de las prácticas finales donde los alumnos ya tenían conocimientos básicos de todo el procesado que incluye un modelo de aprendizaje máquina. Para ello se seleccionaron unos datos para un problema de regresión donde teníamos entradas $X$ y salidas $Y$, y se particionaron estos datos en dos conjuntos: entrenamiento y test. A los alumnos se les proporcionan los datos de entrenamiento tanto la entrada como la salida, y de los datos de test únicamente la entrada. Los alumnos tenían que proporcionar un fichero en formato CSV con las predicciones para los datos de test. Para facilitarles la faena y homogeneizar los resultados se les proporcionó un archivo (notebook) con un ejemplo básico de cómo se debían de leer los datos y guardar las predicciones. Como entrega se pedía que subieran las predicciones de los datos de test al aula virtual junto con el notebook que habían utilizado para hacer las predicciones.

Segundo procedimiento: en examen. En la misma asignatura se propuso realizar un examen de manera similar a lo realizado en prácticas. Se realizó un dataset y se dividió de la misma manera, en entrenamiento y test. Este dataset ha de ser seleccionado cuidadosamente ya que es imprescindible que los alumnos no tengan acceso al dataset original para que no puedan obtener las predicciones. En nuestro caso seleccionamos un dataset que utilizamos internamente para investigación por lo que los alumnos no tenían acceso al dataset original. Del mismo modo se les pidió que subieran las predicciones y el notebook de entrenamiento explicando cada paso realizado y el motivo.

\subsubsection{Control Digital}

En esta sección se muestra un ejemplo de uso de la metodología de hackathon para el aprendizaje de programación en un contexto diferente al de aprendizaje máquina. Concretamente se realizó un hackathon interno en la última sesión de laboratorio de la asignatura Control Digital (Grado en Ingeniería Electrónica Industrial). Los alumnos se organizan por parejas (no pueden repetir con la pareja que han realizado el resto de prácticas) y a cada equipo se le asigna un prototipo distinto del mismo proceso (motor CC con reductora). El modelo paramétrico de cada motor es conocido, de modo que se dispone del ground truth, pero el alumnado no tiene acceso a dicha información.

El objetivo de la competición es que cada grupo haga la identificación del modelo dinámico, realice el diseño del control digital, ajuste los parámetros para cumplir unas determinadas especificaciones e implemente el algoritmo en un microcontrolador para validarlo sobre el proceso real. La puntuación obtenida por cada equipo depende de los siguientes factores: el tiempo en realizar la tarea, la precisión del ajuste del modelo identificado con respecto al del sistema real y el cumplimiento de las especificaciones. Los equipos se clasifican en función de la puntuación obtenida. Para la evaluación se tiene en cuenta el orden de la clasificación, pero también se debe presentar un informe técnico explicando todos los pasos, así como los resultados obtenidos en la identificación paramétrica, el diseño y la validación del algoritmo de control digital. 


\subsection{Nivel 3}

\subsubsection{Big Data}

En la asignatura Big Data (Máster en Ciencia de Datos) pusimos en práctica el nivel 3. Se trata de una asignatura donde se adquieren competencias específicas para el procesamiento de grandes volúmenes de datos y se introducen las plataformas computacionales capaces de manejar estos datos eficientemente. La última parte se orienta al análisis y desarrollo de aplicaciones basadas en aprendizaje máquina sobre plataformas de Big Data o clouds. Para la evaluación de esta última parte, les proponemos la participación en un hackathon externo por equipos (2-3 personas). El hackathon constituye un reto y un problema atractivo al que los estudiantes se enfrentan activamente poniendo en práctica los conocimientos adquiridos en clase en un entorno semi-profesional. Se dedica una sesión específica a la introducción del hackathon donde se analiza el problema y se proporcionan códigos de ejemplo a los estudiantes que les faciliten un primer planteamiento de la solución. En esta sesión el docente propone ideas, trucos y consejos sobre como abordar el problema. Para la evaluación deben entregar su posición en la clasificación oficial del datathon (leaderboard) y un informe de máximo 8 páginas siguiendo una estructura y formato especificado en una plantilla LaTeX. Se proporciona asímismo la rúbrica de evaluación del trabajo, que consiste en:

- Diseño y calidad de la metodología propuesta.

- Interpretación de los modelos y resultados.

- Utilización de las tecnologías explicadas en la asignatura.

Con esta forma de evaluación valoramos que los alumnos integren en su solución los conocimientos adquiridos en la asignatura y balanceamos, por un lado, tener puntuaciones altas en la clasificación del datathon y por otro su capacidad de explicar por qué las soluciones propuestas funcionan (o no). En el curso 2019-2020 se propusieron dos hackatones, uno basado en inundaciones en Malawi ${ }^{3}$, y otro en clasificación de cultivos ${ }^{4}$.

\section{Resultados}

\subsection{Nivel 1}

\subsubsection{Aprendizaje profundo y Aprendizaje máquina II}

El balance de estas actividades suele ser muy positivo. El método permite romper el ritmo de fatiga de una forma muy eficiente. Además, con poco tiempo y esfuerzo se obtiene una puesta en común muy buena entre compañeros y permite al docente remarcar muchas cosas prácticas que en clase resulta más tedioso. Gracias a estos ejercicios se ha reducido drásticamente una crítica recurrente

\footnotetext{
${ }^{3}$ https://zindi.africa/competitions/2030-vision-flood-prediction-in-malawi/data

${ }^{4}$ https://zindi.africa/competitions/iclr-workshop-challenge-2-radiant-earth-computer-vision-for-crop-recogn data
} 
de los alumnos que consistía en su falta de habilidad al enfrentarse a un problema. En particular en las redes neuronales artificiales definir la arquitectura es un proceso que requiere de práctica. $\mathrm{Al}$ ver que ellos obtienen un resultado y además observar cual ha sido el proceso de compañeros para la resolución del ejercicio reducen su aversión a definir y modificar un modelo de red neuronal artificial.

\subsubsection{Análisis y Extracción de la Información}

Planteando la interpretación de las líneas de código de la demo en común, nos fue posible detectar algunas dudas y solucionarlas, y al mismo tiempo, percibimos que los alumnos permanecen especialmente atentos y participativos. Respecto al trabajo en grupo, pudimos apreciar que se iban planteando dudas y ayudando unos a otros hasta llegar a la misma solución, cada uno en su portátil. Mediante la actividad propuesta, los alumnos pudieron implementar directamente muchas de las aproximaciones vistas en teoría y comparar sus resultados con los obtenidos en una publicación científica. Este ejercicio nos permitió además en la puesta en común analizar los resultados obtenidos con aproximaciones diferentes a un mismo problema y debatir acerca de las ventajas y limitaciones que presenta cada una.

\subsection{Nivel 2}

\subsubsection{Aprendizaje máquina}

Los resultados de aplicar le metodología de nivel 2 en la asignatura de aprendizaje máquina (sec. 3.2.1) fueron los siguientes. Con respecto a la experiencia en prácticas, la participación e implicación en esta sesión fue claramente superior a la del resto. Mientras que en las otras sesiones no se recibió ningún correo ni feedback por parte de los alumnos, en esta sesión alrededor de la mitad de los alumnos expresaron de forma activa su compromiso positivo con la tarea.

En cuanto al examen hemos encontrado que esta manera es una forma bastante eficiente de que el alumno se enfrente a una tarea realista. Básicamente se enfrenta a un problema que ha de resolver desde cero. A diferencia de otras asignaturas donde la solución de los problemas suele ser única y, por tanto, un fallo en el procedimiento acarrearía que todo estuviese mal y fallase, en este tipo de asignaturas la aproximación a una solución adecuada se hace incrementando la calidad del modelo. Por ello, este sistema es aplicable en este tipo de problemas en particular, permitiendo la evaluación de todos los procesos explicados en clase realizando únicamente un ejercicio.

\subsubsection{Control Digital}

Este formato de competición se realiza con bastante frecuencia en congresos internacionales de ingeniería de sistemas, automática y robótica, pero no es tan común en la docencia universitaria. A pesar de que una actividad de este tipo es más compleja porque está menos guiada que el resto de laboratorios, el hecho de realizarla al final del curso hace posible que el alumnado tenga mayor dominio de la materia y pueda resolver un problema complejo en un tiempo limitado que en otras condiciones no sería capaz. Además, la competición añade una motivación extra que implica un mayor nivel de concentración y una actitud proactiva para intentar hacerlo lo mejor posible. Dicha actitud no se ha visto en el resto de laboratorios, lo cual indica que introducir elementos y 
actividades de gamificación puede aumentar el rendimiento y la motivación en el aprendizaje de competencias y habilidades de programación.

La metodología del hackathon se puede aplicar en la enseñanza superior de competencias en múltiples áreas STEM (Ciencias, Tecnología, Ingeniería y Matemáticas).

\subsection{Nivel 3}

\subsubsection{Big Data}

La propuesta de participación en un hackathon externo como parte de la evaluación de la asignatura de Big Data supone un desafío no sólo para los estudiantes sinó también para el equipo docente. Primero, porque requiere que alguno de los hackathones activos se ajusten al curriculum de la asignatura. Aunque, como hemos visto anteriormente, existen varias plataformas donde se plantean problemas regularmente éstos han de estar alineados con el temario de la asignatura y con los objetivos docentes. Segundo porque, una vez seleccionado el problema, es necesario analizar y pre-procesar los datos proporcionados en el hackathon para determinar la viabilidad del mismo; el objetivo de la práctica ha de ser que los alumnos sean capaces de resolver el problema propuesto con los conocimientos adquiridos en la asignatura en un tiempo reducido (3-4 semanas de dedicación a tiempo parcial). En tercer lugar, hay que preparar el material específico para la sesión introductoria que facilite una primera aproximación al problema. La preparación naturalmente es específica de un hackaton planteado y por tanto cambia de un año a otro.

Los resultados obtenidos en los hackatones propuestos para el curso 2019-20 fueron muy satisfactorios: todos las trabajos utilizaron de forma extensiva las tecnologías explicadas en la asignatura y todos los trabajos llegaron a enviar soluciones correctas a la web del hackathon. Algunos de ellos, además, hicieron más de 20 envíos a la web del datathon lo que indica el grado de compromiso y dedicación. También cabe destacar la actuación extraordinaria de dos de los equipos: uno de ellos, en el momento de la entrega, iban situados en la posición octava (entre más de 300 equipos); el otro acabó en posición décima al finalizar el datathon.

\subsection{Encuestas de los estudiantes}

Con el fin de reportar evidencias desde lo que ha significado la experiencia de aprendizaje de los estudiantes, se realizaron encuestas a los estudiantes de tres de las asignaturas en las que habíamos introducido la metodología propuesta:

- Aprendizaje máquina 1 (AM I) del Grado en ciencia de datos: una encuesta acerca de su experiencia con el nivel básico (participación $32 \%$ ), y otra encuesta acerca de su experiencia con el segundo nivel (participación 30\%).

- Aprendizaje máquina 2 (AM II) del Máster en ciencia de datos: una encuesta acerca de su experiencia con el nivel básico (participación $41 \%$ ).

- Big data (BD) del Máster en ciencia de datos:, una encuesta acerca de su experiencia con el tercer nivel (participación 41\%). 

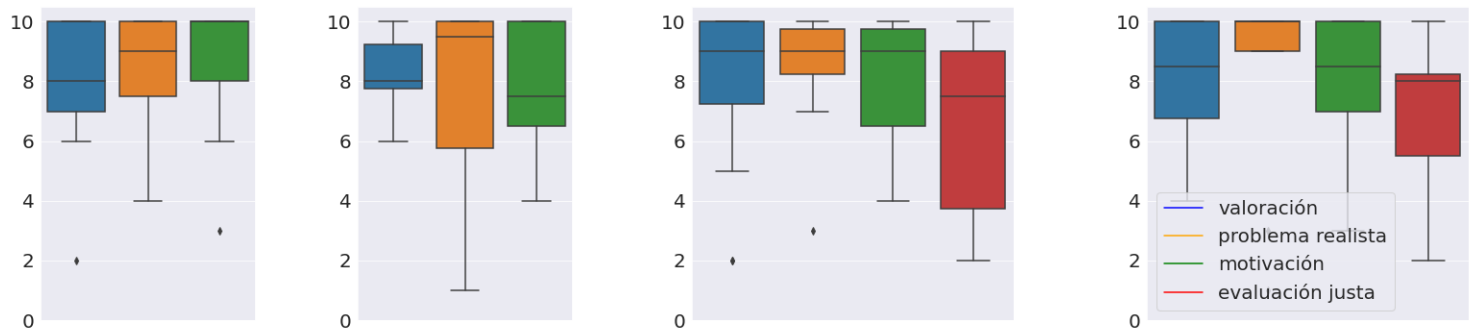

Fig. 1: Resultados de las encuestas realizadas a los alumnos sobre la metodología propuesta. Se realizaron 4 encuestas distintas en tres asignaturas distintas. Cada color representa una de las preguntas realizadas (ver el texto para detalles).

Las encuestas de diseñaron para que fuesen ágiles y nos permitiesen conocer la opinión de los estudiantes respecto a su experiencia de aprendizaje. Los cuestionarios constaban de 3 preguntas con una escala de valoración entre 0 y 10 (4 preguntas en los casos en los que se incluía la evaluación), y una última pregunta abierta donde se les ofreció la posibilidad de incluir un comentario adicional respecto a su experiencia con la actividad. Las preguntas específicas sobre la metodología realizadas en la encuesta fueron:

- Valoración: ¿Cómo valoras esta práctica comparándola con una actividad clásica?

- Problema realista: ¿Piensas que haber participado en este tipo de actividades te prepara mejor para el mundo real?

- Motivación: ¿Te motiva más o menos que una actividad clásica?

- Evaluación justa: ¿Piensas que es una forma justa de evaluar una asignatura?

La figura 1 muestra los resultados de las encuestas. La valoración de la actividad es en general buena en los tres niveles, si bien en el nivel 1 algunos estudiantes destacaron en sus comentarios como puntos negativos el hecho de no haber llegado todos con la misma preparación (no se avisó de la actividad previamente) y que la tarea era quizá demasiado compleja para el tiempo del que se disponía. El hecho de enfrentarse a un problema real end-to-end y a la motivación del aprendizaje se ha valorado positivamente en la mayoría de los casos, y algunos alumnos han destacado en sus comentarios que era su primera experiencia con un datathon. Como dato curioso, un estudiante propone motivar además la competición con algún premio simbólico. En el uso de la metodología para evaluación es donde se han recibido comentarios más críticos, aunque la valoración es también positiva (mediana de 8 sobre 10 en los dos niveles). En el nivel 2 varios alumnos destacan que se dispone de poco tiempo para diseñar soluciones, entrenar modelos y obtener resultados, y que se pueden tener posibles problemas técnicos con librerías, aumentando los nervios y estrés naturales de un examen. Asimismo, varios comentarios hacen referencia a que la nota no debería ir asociada al puesto en el que han quedado los alumnos. Esto no ha sido así en ningún caso, pero pone de manifiesto la necesidad de explicar bien la rúbrica antes del examen. Por contrapartida, un estudiante valora positivamente la posibilidad de tener libertad y creatividad para poner en práctica los conocimientos adquiridos y resolver el problema planteado. En el nivel 3 varios comentarios apuntan a que su experiencia de aprendizaje hubiera sido más completa si después de la entrega 
del trabajo se hubieran comentado en clase los problemas con los que se han encontrado, resolver dudas y mostrar una solución modelo.

\section{Conclusiones}

Programar es difícil porque implica un proceso de transformación de conceptos mentales descritos en términos corrientes a algoritmos descritos en términos compatibles con el ordenador. Este proceso es lento y requiere mucha experiencia práctica y motivación. Por eso, su enseñanza tiene como principal objetivo lograr que los alumnos desarrollen sus capacidades, adquiriendo competencias para crear programas computacionales que resuelvan problemas reales.

En este trabajo hemos propuesto una metodología basada en la idea de hackathon o datathon para reducir los problemas en el proceso de enseñanza-aprendizaje de programación. La metodología propuesta está principalmente enfocada a motivación, pero también puede utilizarse para evaluación. Si bien los ejemplos mostrados se centran en el desarrollo de algoritmos de machine learning, la metodología podría ser ampliamente aplicable en la enseñanza superior de competencias en áreas STEM (Ciencias, Tecnología, Ingeniería y Matemáticas), tal y como se ha demostrado con ejemplos de aplicación en titulaciones técnicas.

La metodología se ha planteado en tres niveles de aplicación del hackathon. El primero consiste en motivar al alumnado a través de su participación en una competición improvisada en el marco de una clase teórica o laboratorio. El segundo nivel consiste en el uso de hackathones programados en la asignatura, los cuales pueden servir tanto para la evaluación continua como para la evaluación final. El tercer nivel implica la participación en un hackathon externo que puede servir como elemento de evaluación o para fomentar el trabajo en equipo resolviendo problemas realistas en un entorno semi-profesional. El hackathon es un desafío de programación limitado en el tiempo por lo que sirve para aprender a trabajar bajo presión, a pensar rápido, y a trabajar en equipo aplicando habilidades transversales y multidisciplinares.

Las encuestas realizadas a los estudiantes muestran que en la mayoría de los casos valoran muy positivamente la experiencia de aprendizaje en términos de motivación y de desarrollo de capacidades para poder abordar un problema realista. La valoración del uso de la metodología para evaluación es también positiva, si bien parece que hay más disparidad de opiniones. Los comentarios específicos recibidos nos ayudarán sin duda a mejorar la aplicación de la metodología en el futuro.

Un camino a seguir dentro de estas metodologías sería aplicar algoritmos que permitiesen evaluar errores en el código entregado por los alumnos de forma automática (Tip y col. 2001). Por otro lado, como trabajo futuro también se plantea extender su aplicación a las asignaturas de Tecnologías de la Información y la Comunicación (TIC) en enseñanzas de magisterio o bellas artes. 


\section{Referencias bibliográficas}

Camps-Valls, G. y col. (2011). Remote sensing image processing. Vol. 5. 1. Morgan \& Claypool Publishers, págs. 1-192.

Dolgopolovas, V. y V. Dagienè (2021). «Computational thinking: Enhancing STEAM and engineering education, from theory to practice». En: Computer Applications in Engineering Education 29.1, págs. 5-11.

Dorn, A. y col. (2020). «Evaluating effectiveness of innovative education formats for 21st century skills: The example of DaVinciLab YouthHackathon Workshops 2019/2020». En: págs. 386-392.

García, Juan Antonio Torrecilla (2020). «Aprendizaje por proyecto en la modalidad hackathon para crear nuevos espacios de desarrollo de competencias transversales entre estudiantes y egresados de la Universidad». En: Innovación Docente e Investigación en Ciencias Sociales, Económicas, y Jurídicas. Avanzando en el proceso de enseñanza-aprendizaje. Dykinson, págs. 1009-1018.

Gardó, H. y J. Riera (2020). «Discovering civic educational hackathons: An exploratory study [Descubriendo las hackatones educativas comunitarias: Un estudio exploratorio]». En: Aloma 38.1, págs. 51-62.

Imbernon, F. (2014). Metodología y dinamización de la enseñanza. Madid:AECI.

Mohan, A. y col. (2010). «Professional Skills in the Engineering Curriculum». En: IEEE Transactions on Education 53.4, págs. 562-571.

Muñoz, Eva Perea, Vanesa Berlanga Silvente y Carme Ruiz Viñals (2017). «Actividades emprendedoras y espacios alternativos de aprendizaje: desarrollo de competencias durante un hackathon day». En: EA, Escuela abierta: revista de Investigación Educativa 20, págs. 47-61.

Porras, J. y col. (2018). «Hackathons in software engineering education: Lessons learned from a decade of events». En: págs. 40-47.

Tip, F. y col. (ene. de 2001). «A Slicing-Based Approach for Locating Type Errors». En: 10.1, págs. 5-55. ISSN: 1049-331X.

Verrelst, J. y col. (2012). «Retrieval of Vegetation Biophysical Parameters Using Gaussian Process Techniques». En: IEEE Transactions on Geoscience and Remote Sensing 50, págs. 1832-1843. 